\title{
Role of Turbulent Prandtl Number on Heat Flux at Hypersonic Mach Numbers
}

\author{
X. Xiao*, J. R. Edwards ${ }^{\dagger}$, and H. A. Hassan ${ }^{\ddagger}$ \\ North Carolina State University, Raleigh, NC 27695-7910 \\ and \\ R. L. Gaffney, Jr. ${ }^{\S}$ \\ NASA Langley Research Center, Hampton, VA 23681-2199
}

\begin{abstract}
A new turbulence model suited for calculating the turbulent Prandtl number as part of the solution is presented. The model is based on a set of two equations: one governing the variance of the enthalpy and the other governing its dissipation rate. These equations were derived from the exact energy equation and thus take into consideration compressibility and dissipation terms. The model is used to study two cases involving shock wave/boundary layer interaction at Mach 9.22 and Mach 5.0. In general, heat transfer prediction showed great improvement over traditional turbulence models where the turbulent Prandtl number is assumed constant. It is concluded that using a model that calculates the turbulent Prandtl number as part of the solution is the key to bridging the gap between theory and experiment for flows dominated by shock wave/boundary layer interactions.
\end{abstract}

\section{Introduction}

Present simulation of turbulent flows involving shock wave/boundary layer interaction invariably over-estimates heat flux by almost a factor of two. ${ }^{1}$ One possible reason for such a performance is a result of the fact that the turbulence models employed make use of Morkovin's hypothesis. ${ }^{2}$ This hypothesis is valid for non-hypersonic Mach numbers and moderate rates of heat transfer. At hypersonic Mach numbers, high rates of heat transfer exist in regions where shock wave/boundary layer interactions are important. For such flows, temperature fluctuations, which are as important as velocity fluctuations at the higher Mach numbers, play a major role in determining wall heat flux and their effects must explicitly be taken into consideration. As a result, one should not expect traditional turbulence models to yield accurate results.

The goal of this investigation is to explore the role of a variable Prandtl number formulation in predicting heat flux in flows dominated by strong shock wave/boundary layer interactions. The intended applications involve external flows in the absence of combustion such as those encountered in supersonic inlets. This can be achieved by adding equations for the temperature (enthalpy) variance and its dissipation rate. Such equations can be derived from the exact Navier-Stokes equations. Traditionally, modeled equations (see, for example, Ref. 3,4) are based on the low speed energy equation where the pressure gradient term and the term responsible for energy dissipation are ignored. It is clear that such assumptions are not valid for hypersonic flows.

The approach used here is based on the procedure used in deriving the $k-\zeta$ model,,${ }^{5}$ in which the exact equations that governed $k$, the variance of velocity, and $\zeta$, the variance of vorticity, were derived and modeled. For the variable turbulent Prandtl number, the exact equations that govern the temperature (enthalpy) variance and its dissipation rate are derived and modeled term by term. The resulting set of equations are free of damping and wall

\footnotetext{
* Research Assistant Professor, Dept. of Mechanical and Aerospace Engineering, Member AIAA

Associate Professor, Dept. of Mechanical and Aerospace Engineering, Associate Fellow AIAA

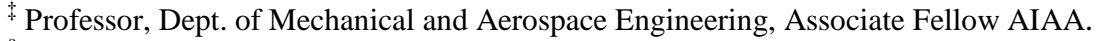

$\S$ Aerospace Engineer, Hypersonic Air Breathing Propulsion Branch, Senior Member AIAA.

Copyright $\bigcirc 2005$ By the American Aeronautics and Astronautics, Inc. All rights reserved.
} 
functions and are coordinate-system independent. Moreover, modeled correlations are tensorially consistent and invariant under Galilean transformation.

Two flat plate experiments are used to determine model constants. The first is the Mach (M) 9.2 experiments of Coleman and Stollery ${ }^{6}$, which was conducted in a hypersonic gun tunnel at Imperial College. The second is the M=8.3 experiments of Kussoy et al. ${ }^{7}$ which were conducted in the Ames 3.5 Foot Hypersonic Wind Tunnel Facility. This turned out to be a major undertaking because of the different facilities and instrumentation and because no accuracy estimates of heat transfer measurement wave provided. In order to put things in proper perspective, it is noted that recent heat transfer measurements ${ }^{8}$ on an elliptic cone in the AEDC Tunnel B estimated uncertainties in excess of $\pm 10 \%$. The model is validated by recent non-intrusive measurements by Schülein ${ }^{9}$ of flows involving shock wave/boundary layer interactions at $M=5$. The measurements were carried out at the Ludwig Tube Facility at DLR. Oil-film interferometry techniques were used to measure skin friction while an infrared camera system was used for heat transfer measurements.

The recent measurements of Schülein were a repeat of an earlier experiment ${ }^{10}$ which did not include heat transfer measurements or skin friction measurements in the separated flow region. Calculations of the earlier experiments were carried out by Nance and Hassan ${ }^{11}$ using a k- $\zeta$ two-equation and an abbreviated stress model. It was concluded in Ref. 10 that there was a need to develop turbulence models capable of predicting the turbulent Prandtl number as part of the solution.

It is shown in this study that a variable Prandtl number formulation results in significant improvement of heat transfer predictions in the presence of shock wave/boundary layer interactions. However, the new model has insignificant influence on wall pressure and skin friction distributions.

\section{Formulation of the Problem}

\section{Governing equations}

The energy equation can be written as

$$
\rho \frac{D h}{D t}=\frac{\partial}{\partial t}(\rho h)+\frac{\partial}{\partial x_{i}}\left(\rho u_{i} h\right)=\frac{D p}{D t}-\frac{\partial q_{i}}{\partial x_{i}}+\phi \equiv S_{h}
$$

where $\quad q_{i}=-\lambda \frac{\partial T}{\partial x_{i}}$

$$
\begin{aligned}
& \phi=t_{i j} \frac{\partial u_{i}}{\partial x_{i j}} \\
& t_{i j}=2 \mu S_{i j}-\frac{2}{3} \delta_{i j} \frac{\partial u_{m}}{\partial x_{m}} \\
& S_{i j}=\frac{1}{2}\left(\frac{\partial u_{v}}{\partial x_{j}}+\frac{\partial u_{j}}{\partial x_{i}}\right)
\end{aligned}
$$

and $\rho$ is the density, $h$ is the enthalpy, $p$ is the pressure, $u_{i}$ is the velocity and $\lambda$ and $\mu$ are the coefficients of thermal conductivity and molecular viscosity. Noting that

$$
\frac{D P}{D t}=R \rho \frac{D T}{D t}+R T \frac{D \rho}{D t}
$$

and using the relation $h=C_{P} T$ and the conservation of mass equation, Eq. (1) can be re-written as 


$$
\rho \frac{D h}{D t}=-(\gamma-1) \rho h \frac{\partial u_{i}}{\partial x_{i}}+\gamma\left(-\frac{\partial q_{i}}{\partial x_{i}}+\phi\right), \quad \gamma=C_{P} / C_{v}
$$

where $C_{P}$ and $C_{v}$ are the specific heats at constant pressure and constant volume.

The mean energy equation follows from Eq. (1) as

$$
\frac{\partial}{\partial t}(\bar{\rho} \tilde{h})+\frac{\partial}{\partial x_{i}}\left(\bar{\rho} \tilde{h} \tilde{u}_{j}\right)=\frac{D \bar{P}}{D t}-\frac{\partial \bar{q}_{i}}{\partial x_{i}}+\bar{\phi}-\frac{\partial}{\partial x_{j}}\left(\bar{\rho} \overline{h^{\prime \prime} u_{j}^{\prime \prime}}\right)
$$

where

$$
\bar{\phi}=\bar{t}_{i j} \frac{\partial \tilde{u}_{i}}{\partial x_{j}}+\bar{\rho} \in ; \quad \in=v \zeta
$$

where $v$ is the kinematic viscosity and $\zeta$ is the enstrophy.

Equation (4) was the starting point for deriving an equation for the enthalpy variance and its dissipation rate. The exact equations are given in the Appendix, while the modeled equations are given as:

$$
\begin{aligned}
\frac{\partial}{\partial t}\left(\rho \tilde{h}^{\prime \prime 2} / 2\right)+\frac{\partial}{\partial x_{j}}\left(\bar{\rho} \tilde{u}_{j} \tilde{h}^{\prime \prime 2} / 2\right) & =\frac{\partial}{\partial x_{j}}\left[\rho\left(\gamma \alpha+\alpha_{t} C_{h, 2}\right) \frac{\partial\left(\tilde{h}^{\prime \prime 2} / 2\right)}{\partial x_{j}}\right] \\
& +2 \mu \gamma \bar{S}_{i j}\left[\frac{\partial}{\partial x_{j}}\left(q_{t, i} / \rho\right)+\frac{\partial}{\partial x_{i}}\left(q_{t, j} / \rho\right)\right]-\frac{4}{3} \mu \gamma \tilde{S}_{k k} \frac{\partial}{\partial x_{j}}\left(q_{t, j} / \rho\right) \\
& -(\gamma-1) \bar{\rho} \tilde{h}^{\prime \prime 2} \frac{\partial \tilde{u}_{i}}{\partial x_{i}}-q_{t, i} \frac{\partial \tilde{h}}{\partial x_{i}}+2 C_{h, 4} \gamma \mu \sqrt{\tilde{h}^{\prime \prime 2}} \zeta-\gamma \bar{\rho} \in_{h}
\end{aligned}
$$

where

$$
\begin{aligned}
q_{t, j} & =-\bar{\rho} \alpha_{t} \frac{\partial \tilde{h}}{\partial x_{j}} \\
\alpha_{t} & =0.5\left[C_{h} k \tau_{h}+v_{t} / 0.89\right] \\
\tau_{h} & =\tilde{h}^{\prime \prime 2} / \epsilon_{h} \\
& \overline{\epsilon_{h}}=\alpha\left(\frac{\partial h^{\prime \prime}}{\partial x_{i}}\right)^{2}
\end{aligned}
$$

and where $\epsilon_{h}$ is the rate of dissipation of the enthalpy variance, $\alpha$ is the diffusivity, and

$$
v_{t}=C_{\mu} k^{2} / \nu \zeta \equiv C_{\mu} k \tau_{k}
$$

is the turbulent kinematic viscosity. 
The modeled equation for the dissipation of enthalpy variance is

$$
\begin{aligned}
\frac{\partial}{\partial t}\left(\bar{\rho} \epsilon_{h}\right)+\frac{\partial}{\partial x_{j}}\left(\bar{\rho} \tilde{u}_{j} \in_{h}\right) & =C_{h, 3} \alpha-\bar{\rho} \epsilon_{h}\left(C_{h, 5} b_{j k}-\frac{\delta_{j k}}{3}\right) \frac{\partial \tilde{u}_{i}}{\partial x_{k}} \\
& +C_{h, 6} \bar{\rho} k \frac{\partial \sqrt{\tilde{h}^{\prime \prime 2}}}{\partial x_{j}} \frac{\partial \tilde{h}}{\partial x_{j}}+\frac{\partial}{\partial x_{j}}\left[\left(\gamma \alpha+C_{h, 7} \alpha_{t}\right) \frac{\partial \epsilon_{h}}{\partial x_{j}}\right] \\
& +C_{h, 8} \frac{q_{t, j}}{\tau_{h}} \frac{\partial h}{\partial x_{j}}-\gamma \rho \in_{h}\left[\frac{C_{h, 9}}{\tau_{h}}+\frac{C_{h, 10}}{\tau_{k}}\right] \\
& +0.5 C_{h, 11}\left[\sqrt{\tilde{h}^{\prime \prime 2}} / \tau_{h}+k / \tau_{k}\right] \cdot \max \left(\frac{D P}{D t}, 0.0\right)
\end{aligned}
$$

with

$$
b_{j k}=\frac{\tau_{i j}}{\bar{\rho} k}+\frac{2}{3} \delta_{i j}, \quad \tau_{i j}=-\bar{\rho} \overline{u_{i}^{\prime \prime} u_{j}^{\prime \prime}}
$$

The constants, $\mathrm{C}_{\mathrm{h}}, \ldots \mathrm{C}_{\mathrm{h}, 11}$ are model constants and are given in Table 1. The turbulent Prandtl number is defined as

$$
\operatorname{Pr}_{t}=v_{t} / \alpha_{t}
$$

The choice of $\alpha_{t}$ merits further elaboration. It was indicated in Ref. 4 that experiments in simple shear flows showed that the appropriate time scale for temperature fluctuations is proportional to the average of $\tau_{h}$ and $\tau_{k}$. This is the basis for the modeling indicated in Eq. 8. It should be noted that, traditionally ${ }^{3,4}$, the time scale of temperature fluctuations is taken as the geometric average of $\tau_{h}$ and $\tau_{k}$.

\section{Results and Discussion}

The predictions of the model will be compared first with the 15 deg. ramp experiment of Coleman and Strollery. In this experiment no flow separation was indicated. The remaining comparisons will be made with Schülein twodimensional flow measurements for shock angles $\beta$ of 10 and 14 degrees. Flow separation was observed for both of these angles.

The free stream conditions for the experiments of Coleman and Strollery are: $\mathrm{M}=9.2, \mathrm{Re}=47 \times 10^{6} / \mathrm{m}, \mathrm{T}_{0}=$ $1070 \mathrm{~K}, \mathrm{~T}_{\infty}=64.5 \mathrm{~K}$ and $\mathrm{T}_{\mathrm{w}}=295 \mathrm{~K}$.It has been shown in Ref. 11 that use of $241 \times 141$ Cartesian grid with constant spacing in the $x$ (flow) direction and geometric spacing in the $y$ (normal) direction resulted in a grid resolved solution and this grid is employee in the present calculations. Figures 1 and 2 compare the pressure distribution for constant and variable Prandtl number calculations. As is seen from Fig. 1 the pressure distribution is essentially independent of the turbulent Prandtl number. Both constant and variable turbulent Prandtl number calculations overpredict the heat flux in the pressure rise region. The variable turbulent Prandtl number calculation is in good agreement with experiment in the recovery region while the constant Prandtl number calculations underpredict the heat flux in this region.

The experimental setup of the Schülein experiment is shown in Fig. 3. A shock generator is mounted on the upper wall and the resultant oblique shock wave interacts with the turbulent boundary layer growing on the flat plate along the lower wall. The free-stream conditions in the test section were: $M=5$, unit Reynolds number $=37 \times$ $106 / \mathrm{m}, \mathrm{T}_{0}=410 \mathrm{~K}, \mathrm{P}_{0}=2.12 \mathrm{MPa}$ and a wall temperature of $300 \pm 5 \mathrm{~K}$. It was shown in Ref. 11 that a $141 \times 141$ Cartesian grid with constant spacing in the $x$-direction and geometric spacings in the $y$-direction resulted in a grid resolved solution. A grid of $151 \times 141$ is used in this investigation.

The calculations were limited to a region ahead of the point where the reflected shock impinges on the upper surface. This assumption makes it possible to use an extrapolation boundary condition at the outflow. Without this 
assumption, one would be forced to consider the upper wall in the calculations. As is seen below, this assumption affected the solution in an adverse manner near the outflow boundary.

Figures 4-6 compare predictions of surface pressure, wall shear stress and wall heat flux while Figs. 7 and 8 compare temperature and velocity at $x=576 \mathrm{~mm}$ for $\beta=10 \mathrm{deg}$. It is seen from Figs. 4 and 5 that the results are almost identical for both constant and variable turbulent Prandtl numbers. Both calculations underpredict the pressure in the separated region.

The oil-film interferometry technique cannot be used to determine the extent of the separated region. Instead, conventional oil-film visualization was used to deduce the start and end of the separated region. It is seen from Fig. 5 that the extent of separation is well predicted. However, some discrepancies are noted in predicting the wall shear stress in the region. The behavior near the outflow boundary is a result of locating the outflow boundary ahead of the point where the reflected shock impinges on the upper boundary. The flow was responding to the compression resulting from the reflected shock at the upper wall which is responsible for the increase in wall shear stress. On the other hand the calculations were responding to an expanding flow which results in a decrease in shear stress.

Figure 6 shows that constant Prandtl number calculations overpredict peak heating by a factor of 2. Although variable Prandtl number calculations represent a major improvement in estimating the magnitude of the heat flux, the choice of the downstream boundary condition appears to have a major influence on heat transfer predictions downstream of the separated region. Small unsteadiness was noticed in the calculations but the discrepancy cannot be a direct result of that. The fact that heat transfer predictions in the recovery region of the 15 degree ramp were in good agreement with experiment suggest that the outflow boundary condition is partially responsible for the discrepancy.

Figures 7 and 8 show that both temperature and velocity profile are in fair to good agreement with experiment. The fact that temperature distribution for the variable Prandtl number is in better agreement with experiment is a direct result of the fact that the variable Prandtl number formulation results in more realistic heat flux estimates.

Figures 9-13 compare similar prediction for the $\beta=14$ degree case. Similar remarks can be made regarding this case. Experimental measurements show more oscillations in the data and this is reflected in the computations.

\section{Concluding Remarks}

A new approach has been developed for calculating the turbulent Prandtl number as part of the solution. The approach is based on a two-equation model for the enthalpy variance and its dissipation rate. All of the correlations that appear in the exact equation that govern the enthalpy variance and its dissipation rate are modeled in order to ensure the incorporation of relevant physics into the model equations.

The new formulation is used to study flows characterized by shock wave/boundary layer interactions. In general heat flux calculations showed dramatic improvements while surface pressures and wall shear stress were unaffected by the variable Prandtl number formulation.

The discrepancy in the heat flux calculations of the reflected shock experiments of Schülein when the turbulent Prandtl number formulation is employed can be a result of the outflow boundary condition employed, the numerical procedure, a modeling issue, or a combination of all of the above. In spite of the discrepancy, the fact remains, however, that the key to bridging the gap between theory and experiment in flows dominated by shock wave/boundary layer interactions is to use a variable Prandtl number formulation.

\begin{tabular}{|c|c|c|c|c|c|c|c|c|c|}
\hline Ch & Ch,2 & Ch,4 & Ch,5 & Ch,6 & Ch,7 & Ch,8 & Ch,9 & Ch,10 & Ch,11 \\
\hline 0.0648 & 0.5 & -0.4 & $0-0.5$ & -0.12 & 1.45 & 0.7597 & 0.87 & 0.25 & 0.775 \\
\hline
\end{tabular}

\section{Acknowledgment}

The authors would like to acknowledge partial support under NASA Grant NAG1-03030 and Air Force Contract FA9101-04-C-0015/

\section{References}

${ }^{1}$ Thivet, F., Knight, D. D., Zheltovodov, A. A., and Maksimov, A. I., "Insights in Turbulence Modeling for Crossing-Shock Wave/Boundary-Layer Interactions," AIAA Journal, Vol. 39, No. 6, 2001, pp. 985-995.

${ }^{2}$ Morkovin, M., "Effects of Compressibility on Turbulent Flows," Mecanique de le Turbulence, edited by A. Favre, Gordon and Breach, New York, 1964, pp. 367-380.

${ }^{3}$ Sommer, T. P., So, R. M. C., and Zhang, H. A., "Near-Wall Variable-Prandtl-Number Turbulence Model for Compressible Flows," AIAA Journal, Vol. 31, No. 1, 1993, pp. 27-35. 
${ }^{4}$ Abe, K., Kondoh, T., and Nagano, Y., "A New Turbulence Model for Predicting Flow and Heat Transfer in Separating and Reattaching Flows - II. Thermal Field Calculations," International Journal of Heat and Mass Transfer, Vol. 38, No. 4, 1995, pp. 1467-1481.

${ }^{5}$ Robinson, D. F. and Hassan, H. A., "Further Development of the k- $\zeta$ (Enstrophy) Turbulence Closure Model," AIAA Journal, Vol. 36, No. 10, 1998, pp. 1825-1833.

${ }^{6}$ Coleman, G. G. and Stollery, J. L., "Heat Transfer from Hypersonic Turbulent Flow at a Wedge Compression Corner," Journal of Fluid Mechanics, Vol. 56, No. 4, 1972, pp. 741-752.

${ }^{7}$ Kussoy, M. I., Horstman, K. C., and Horstman, C. C., "Hypersonic Crossing Shock-Wave/Turbulent Boundary Interactions," AIAA Journal, Vol. 31, No. 12, 1993, pp. 2197-2203.

8Kimmel, R. L., Poggie, J., and Schwoerke, S. N., "Laminar-Turbulent Transition in a Mach 8 Elliptic Cone Flow," AIAA Journal, Vol. 37, No. 9, 1999, pp. 1080-1087.

${ }^{9}$ Schülein, E., "Optical Skin Friction Measurements in Short-Duration Facilities," AIAA Paper 2004-2115, June 2004.

${ }^{10}$ Schülein, E., Krogmann, P., and Stanwsky, E., "Documentation of Two-Dimensional Impinging Shock/Turbulent Boundary Layer Interaction Flows," DLR 1 B 223-96 A 49, October 1996.

${ }^{11}$ Nance, R. P. and Hassan, H. A., "Turbulence Modeling of Shock-Dominated Flow with a $k-\zeta$ Formulation," AIAA Paper 99-0153, January 1999.

\section{Appendix}

Exact Equations for Enthalpy Variance and its Dissipation Rate

$$
\frac{\partial}{\partial t}\left(\bar{\rho} \tilde{h}^{\prime \prime} / 2\right)+\frac{\partial}{\partial x_{j}}\left[\bar{\rho} \tilde{u}_{j} \tilde{h}^{\prime \prime} / 2\right]=-\overline{\rho h^{\prime \prime} u_{j}^{\prime \prime}} \frac{\partial \tilde{h}}{\partial x_{j}}-\frac{\partial}{\partial x_{j}}\left[\overline{\rho u_{j}^{\prime \prime} h^{\prime \prime 2} / 2}\right]+\overline{h^{\prime \prime} S_{k}}
$$

where

$$
\begin{aligned}
& \overline{h^{\prime \prime} S_{h}}=\overline{h^{\prime \prime} \frac{D p}{D t}}-\overline{h^{\prime \prime} \frac{\partial \xi_{i}}{\partial x_{i}}}+\overline{h^{\prime \prime} \phi} \\
& \rho \frac{D}{D_{t}} \varepsilon_{h}+\overline{2 \rho \alpha \frac{\partial h^{\prime \prime}}{\partial x_{j}} \frac{\partial h}{\partial x_{k}}} \frac{\partial \tilde{u}_{j}}{\partial x_{k}}+\overline{2 \rho \alpha \frac{\partial u_{j}^{\prime \prime}}{\partial x_{k}} \frac{\partial h^{\prime \prime}}{\partial x_{k}}} \frac{\partial \tilde{h}}{\partial x_{j}}+\overline{2 \rho \alpha u_{j}^{\prime \prime} \frac{\partial h^{\prime \prime}}{\partial x_{k}}} \frac{\partial^{2} \tilde{h}}{\partial x_{j} \partial x_{k}} \\
& +\overline{u_{j}^{\prime \prime}\left[\rho \alpha\left(\frac{\partial h^{\prime \prime}}{\partial x_{k}}\right)^{2}\right]}+\overline{2 \rho \alpha \frac{\partial u_{j}^{\prime \prime}}{\partial x_{k}} \frac{\partial h^{\prime \prime}}{\partial x_{k}} \frac{\partial h^{\prime \prime}}{\partial x_{j}}} \\
& =\overline{2 \rho \alpha \frac{\partial h^{\prime \prime}}{\partial x_{k}} \frac{\partial}{\partial x_{k}}\left[\frac{S_{h}^{\prime}}{\rho}-\frac{1}{\rho} \frac{\partial}{\partial x_{j}}\left(\overline{\rho u_{j}^{\prime \prime} h^{\prime \prime}}\right)\right]}
\end{aligned}
$$

where

$$
S_{h}^{\prime}=-\frac{\partial q_{i}}{\partial x_{i}}+\phi^{\prime}
$$




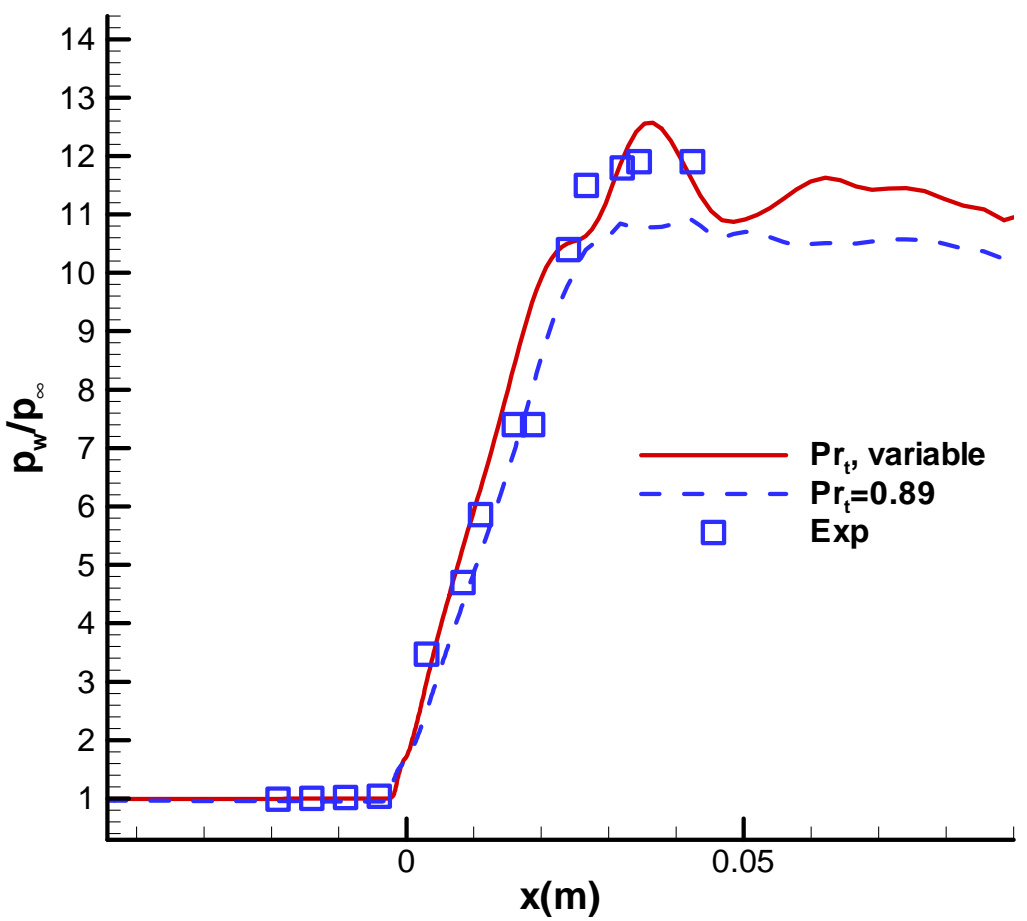

Fig.1 Computed and measured pressure distribution, 15 deg ramp

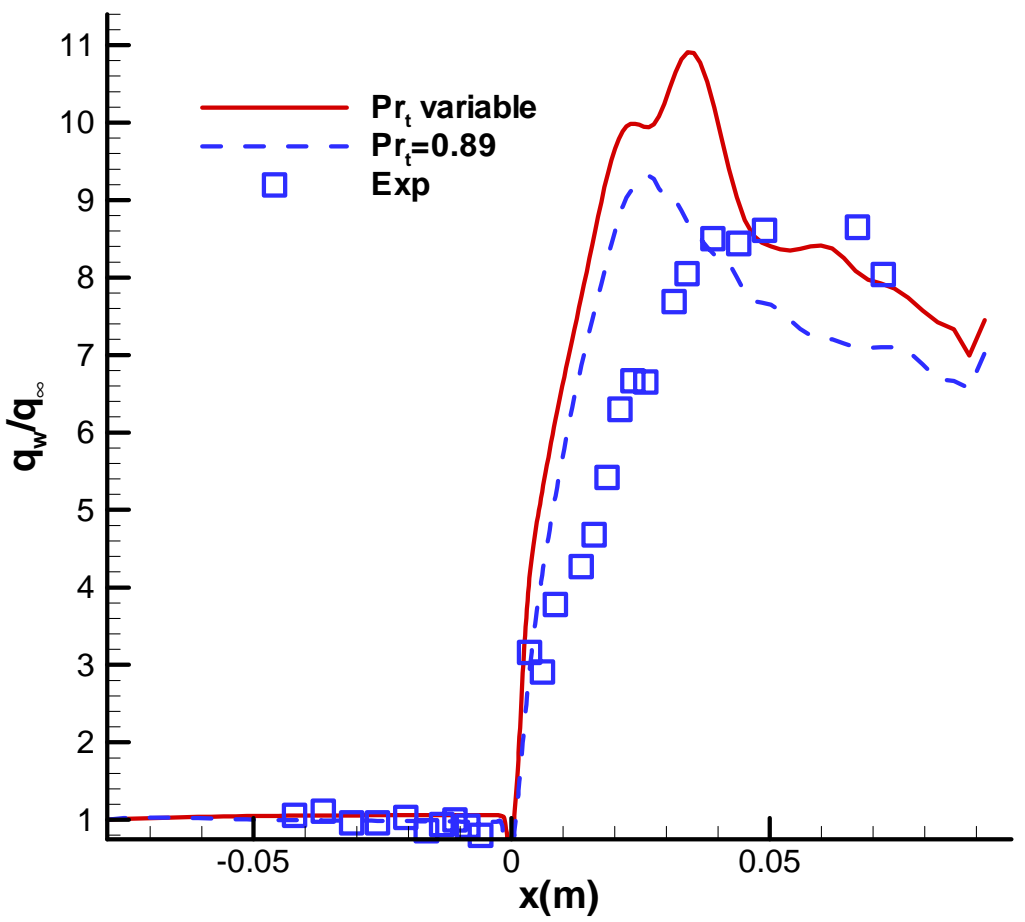

Fig.2 Computed and measured heat flux, 15 deg ramp

American Institute of Aeronautics and Astronautics 


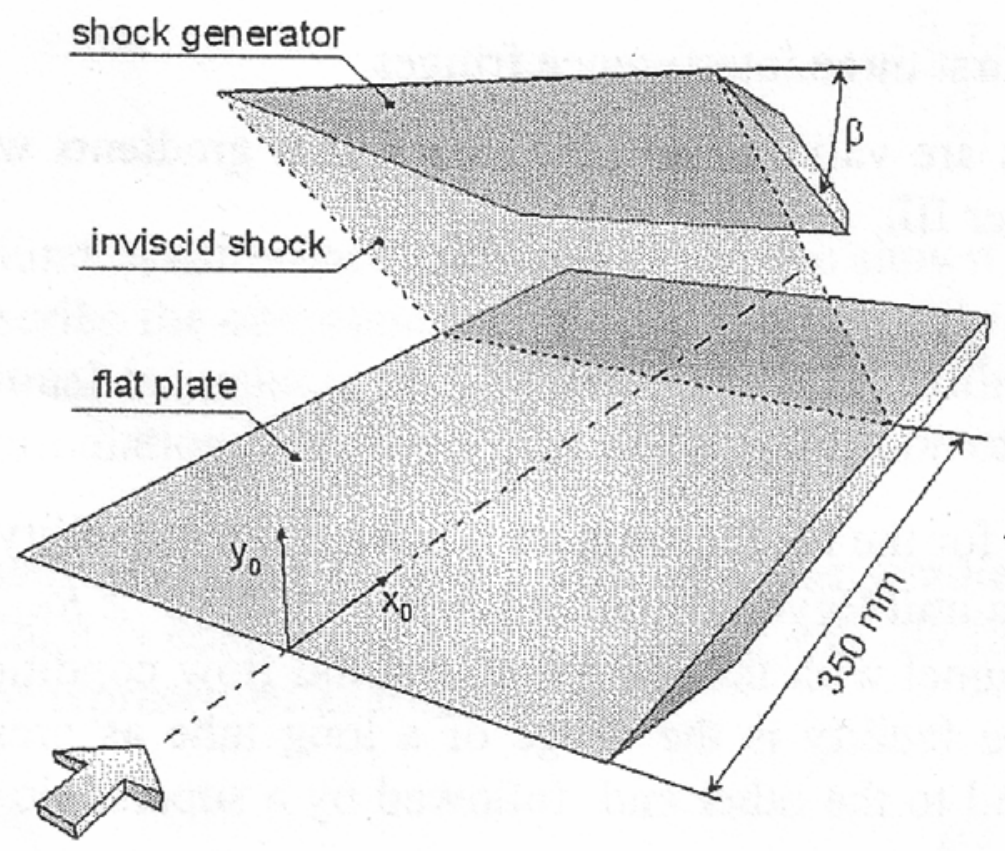

Fig.3 Schematic of shock-wave/boundary layer interaction experiment.

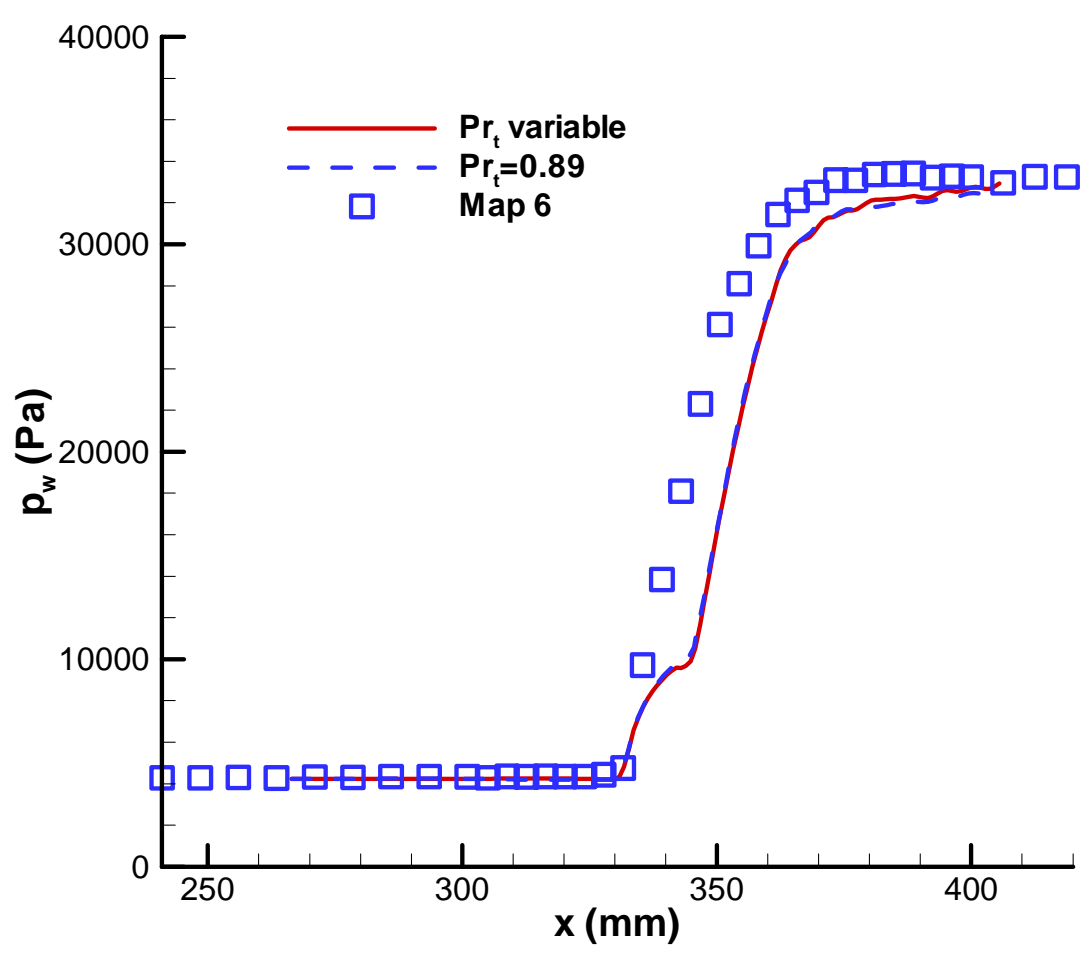

Fig.4 Computed and measured wall pressure, $\beta=10 \mathrm{deg}$ 


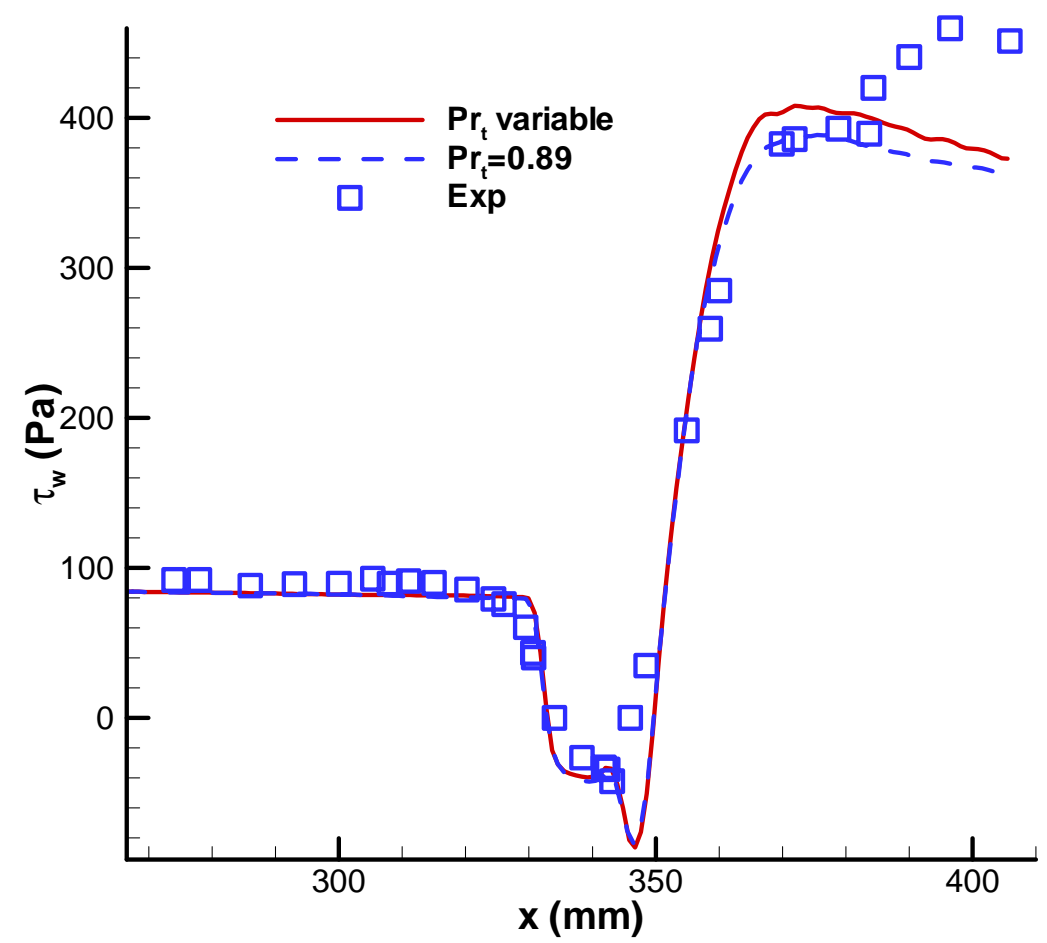

Fig. 5 Computed and measured wall shear stress, $\beta=10 \mathrm{deg}$

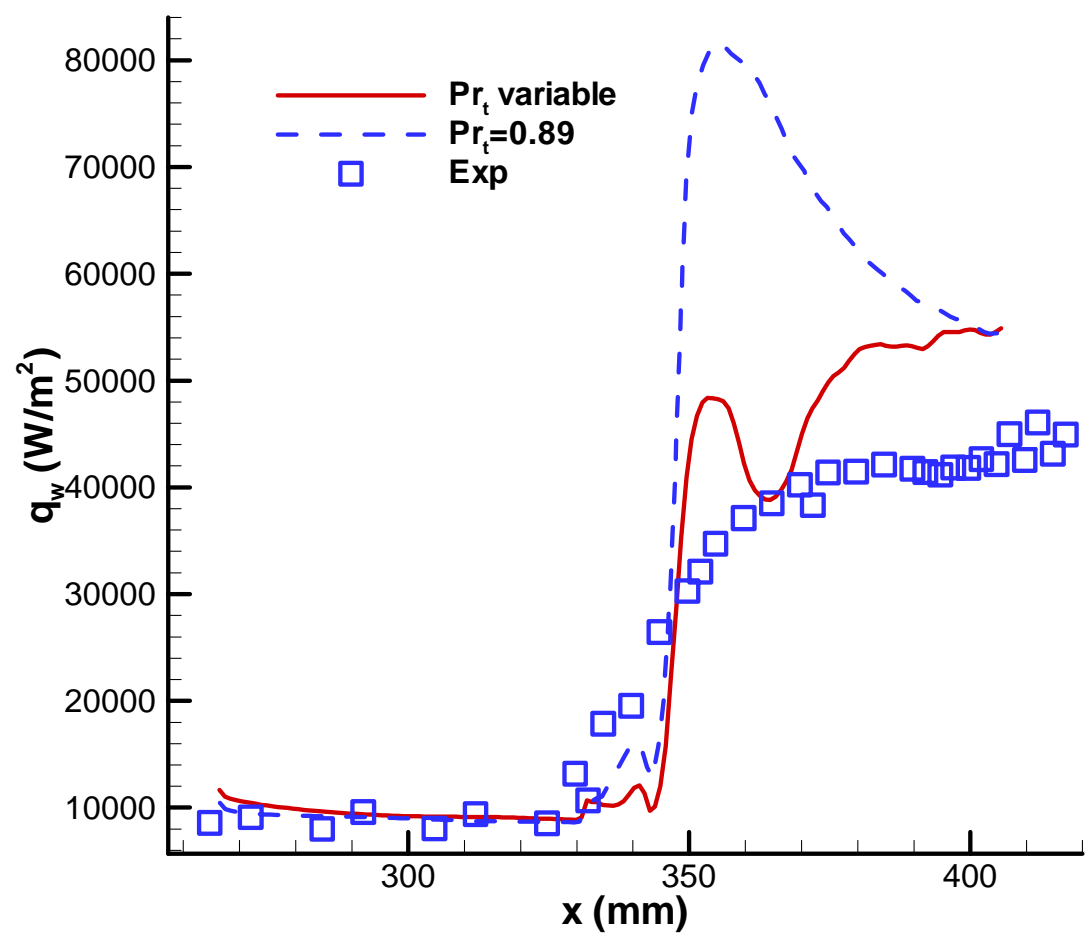

Fig.6 Computed and measured wall heat flux, $\beta=10 \mathrm{deg}$

American Institute of Aeronautics and Astronautics 


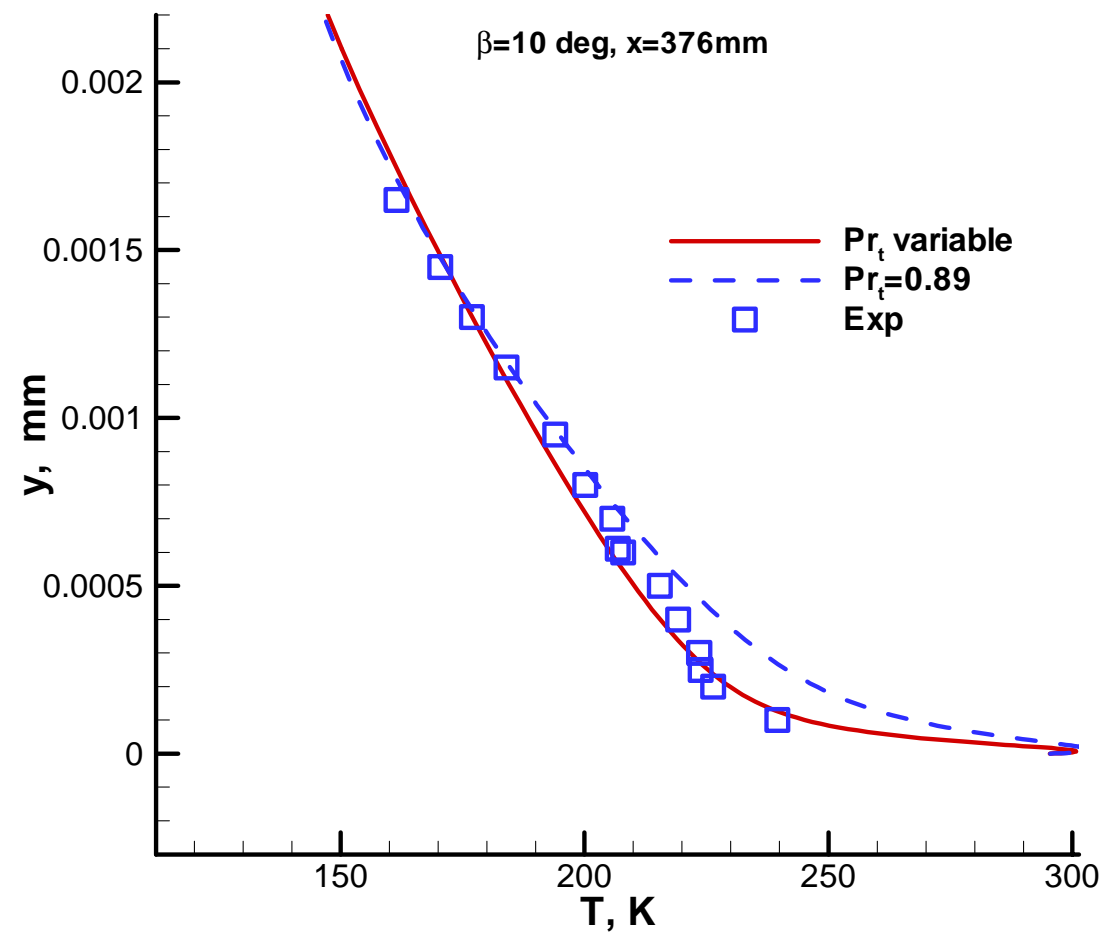

Fig. 7 Computed and measured temperature profile at $x=376 \mathrm{~mm}, \beta=10 \mathrm{deg}$

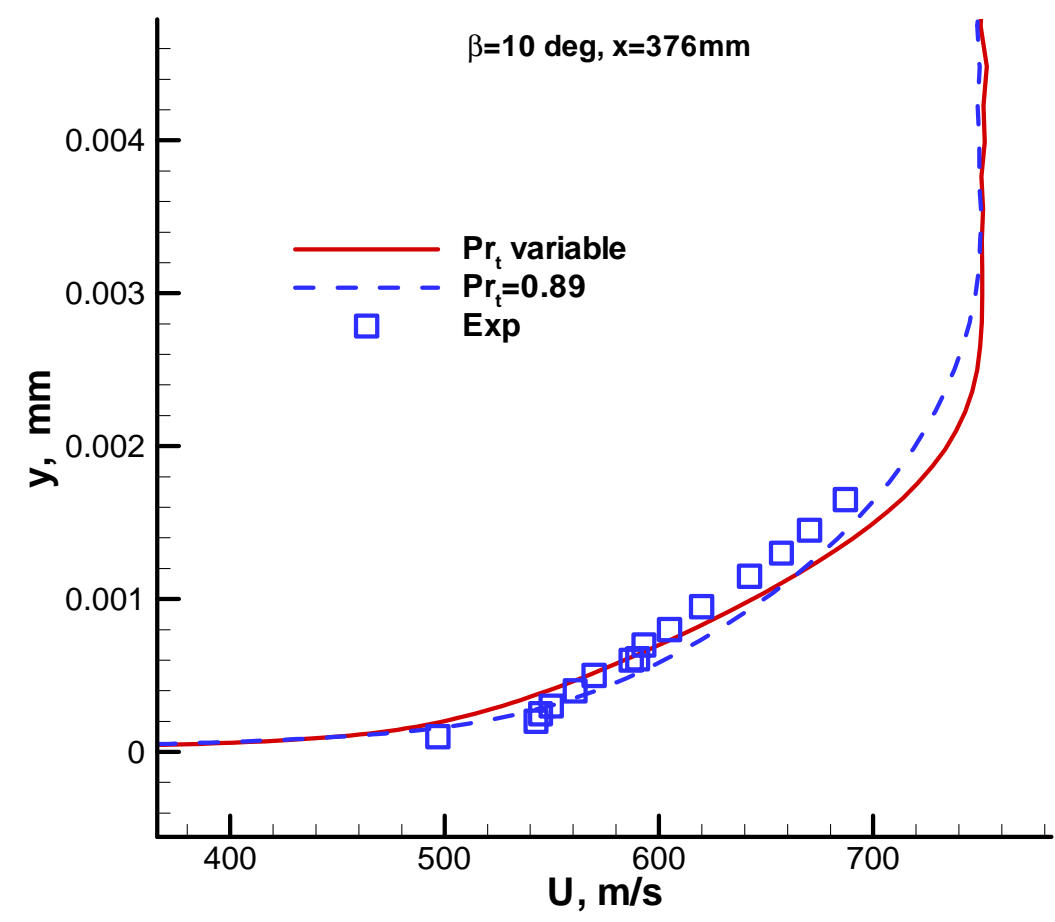

Fig. 8 Computed and measured velocity profile at $x=376 \mathrm{~mm}, \beta=10 \mathrm{deg}$ 10

American Institute of Aeronautics and Astronautics 


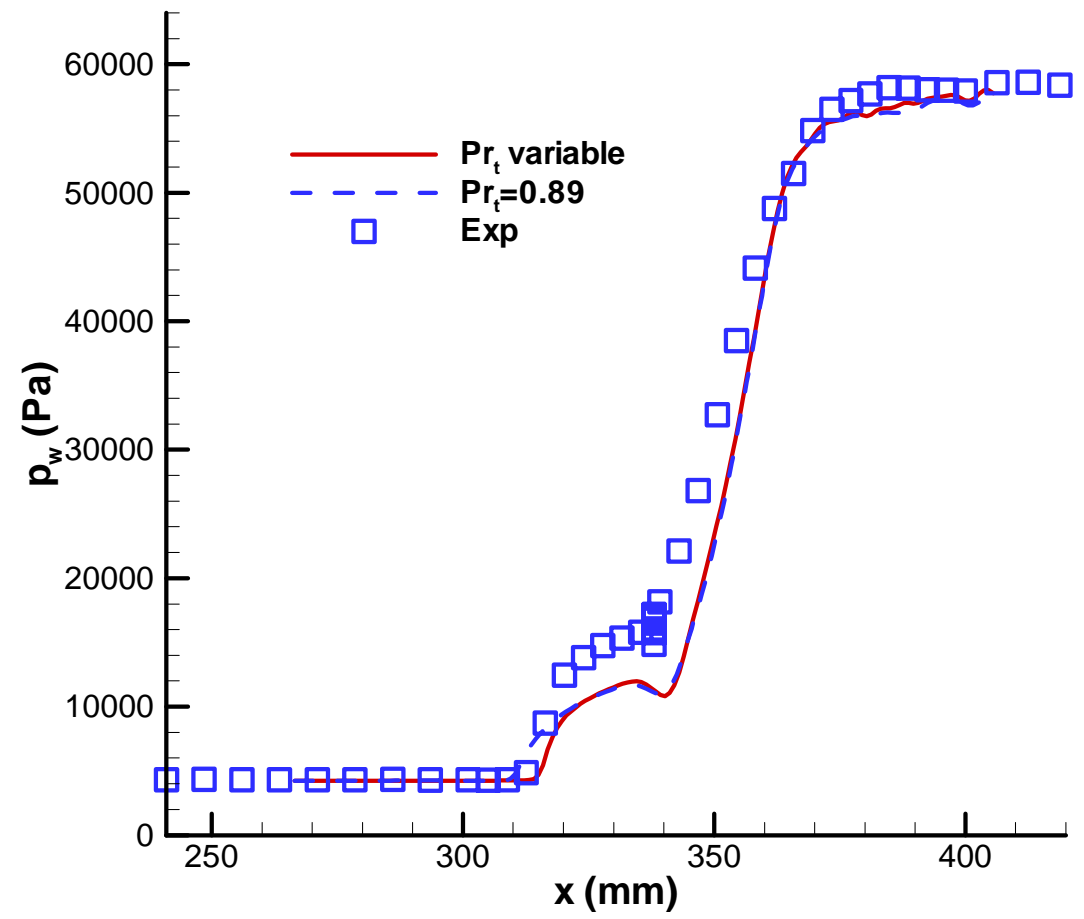

Fig.9 Computed and measured wall pressure, $\beta=14$ deg

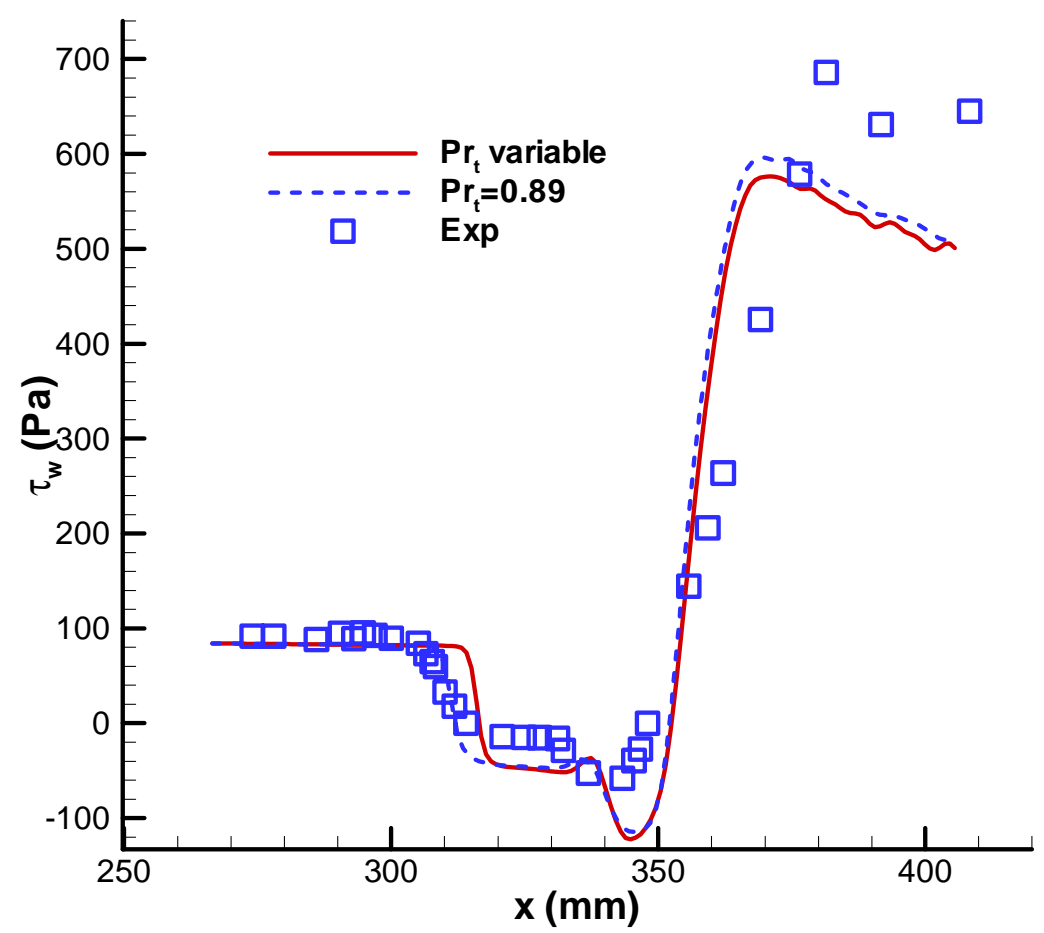

Fig.10 Computed and measured wall shear stress, $\beta=14$ deg

American Institute of Aeronautics and Astronautics 


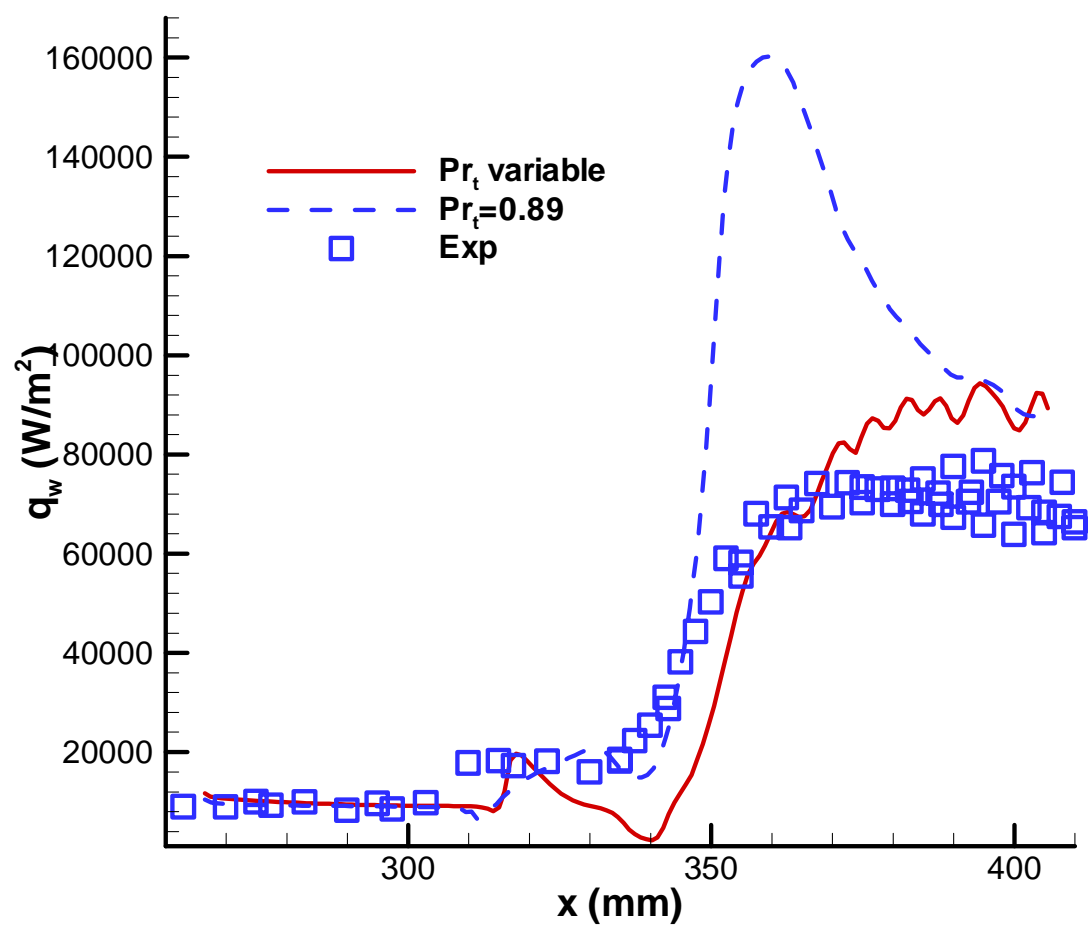

Fig.11 Computed and measured wall heat flux, $\beta=14 \mathrm{deg}$

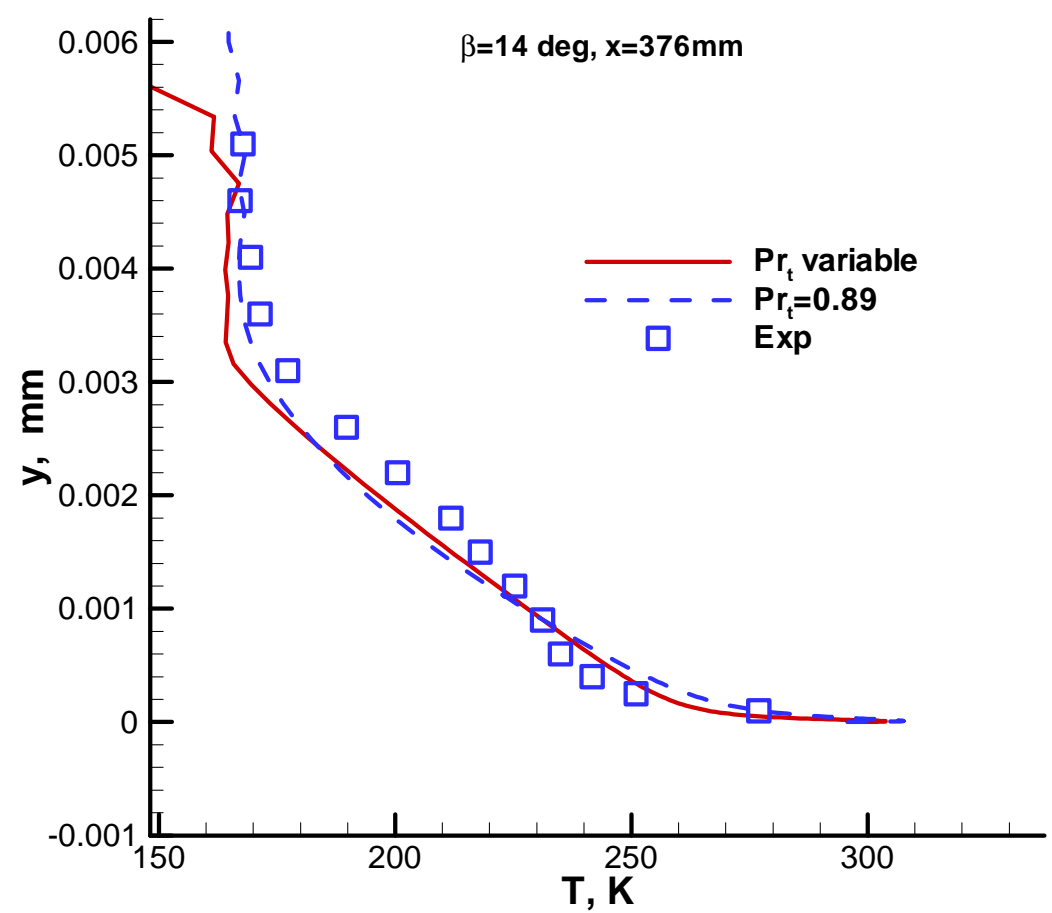

Fig.12 Computed and measured temperature profile, at $x=376 \mathrm{~mm}, \beta=14 \mathrm{deg}$ 


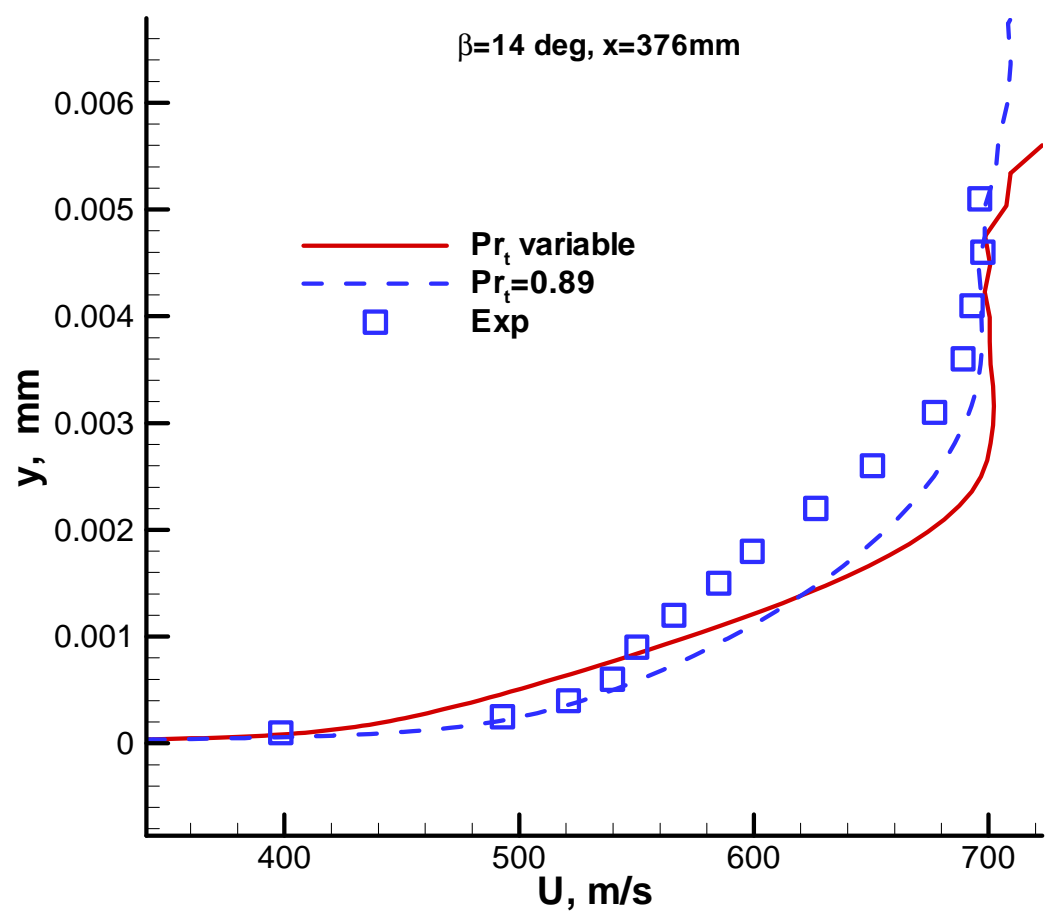

Fig. 13 Computed and measured velocity profile, at $x=376 \mathrm{~mm}, \beta=14 \mathrm{deg}$ 